\title{
Validation of bidimensional measurement in nasopharyngeal carcinoma
}

\author{
Ting-Shou Chang ${ }^{1}$, Sau-Tung Chu' ${ }^{1}$ Yu-Yi Hou' ${ }^{1}$ Kuo-Ping Chang ${ }^{1}$, Chao-Chuan Chi', Ching-Chih Lee ${ }^{2,3^{*}}$
}

\begin{abstract}
Background: Our previous study showed a close relationship between computed tomography (CT)-derived bidimensional measurement of primary tumor and retropharyngeal nodes (BDMprn) and gross tumor volume of primary tumor and retropharyngeal nodes (GTVprn) in nasopharyngeal carcinoma (NPC) and better prognosis for NPC patients with smaller BDMprn. In this study, we report the results on of a study to validate the use of BDM in a separate cohort of NPC patients.
\end{abstract}

Methods: We retrospectively reviewed 103 newly diagnosed NPC cases who were treated with radiotherapy/ concurrent chemoradiotherapy (CCRT) or CCRT with adjuvant chemotherapy from 2002 to 2009. We used magnetic resonance imaging (MRI) to measure BDMprn. We calculated overall survival, recurrence-free and distant metastasisfree survival curves and set a BDMprn cut off point to categorize patients into a high- or low-risk group. We then used Cox proportional hazard model to evaluate the prognostic influence of BDMprn after correcting age, gender and chemotherapy status.

Results: After adjusting for age, gender, and chemotherapy status, BDMprn remained an independent prognostic factor for distant metastasis [Hazard ratio $(H R)=1.046 ; P=0.042$ ] and overall survival ( $H R=1.012 ; P=0.012$ ). Patients with BDMprn $<15 \mathrm{~cm}^{2}$ had a greater 3-year overall survival rate than those with BDMprn $\geqq 15 \mathrm{~cm}^{2}(92.3 \%$ vs. $73.7 \% ; P=0.009$ ). They also had a greater 3 -year distant metastasis-free survival (94\% vs.75\%; $P=0.034$ ).

Conclusion: The predictive ability of BDMprn was validated in a separate NPC cohort. A BDMprn of $15 \mathrm{~cm}^{2}$ can be used to separate NPC patients into high- and low-risk groups and predict survival rates and metastasis potential. It can, therefore, be used as a reference to design clinical trials, predict prognosis, and make treatment decisions.

\section{Background}

Nasopharyngeal carcinoma (NPC) is common among Asians, especially in southern China. While the annual incidence in Western countries is $<1$ per 100,000 population, it is 6.17 per 100,000 in Taiwan [1]. Because it is difficult to approach nasopharyngeal tumors surgically, chemoradiotherapy or radiotherapy is the primary means of treating this disease [2]. The American Joint Committee of Cancer (AJCC) staging system for NPC is widely used to prognosticate and plan for its treatment and is well-accepted as an evaluation tool in clinical research. However, because the current TNM staging approach is limited in its ability to predict prognosis

\footnotetext{
* Correspondence: ml2406@hotmail.com

${ }^{2}$ Department of Otolaryngology, Buddhist Tzu Chi Dalin General Hospital,

Chiayi County 622, Taiwan

Full list of author information is available at the end of the article
}

based on NPC tumor stage [3,4], other factors might be incorporated to further refine prognostic accuracy.

Gross tumor volume is one factor closely related to NPC survival [5-8]. It is not, however, widely advocated as a prognostic factor probably because measuring tumor volume can be time-consuming and labor-intensive. Several studies have used unidimensional and bidimensional measurement to evaluate the tumor size [9-11]. In a previous study, we found bidimensional measurement of primary NPC tumor and retropharyngeal nodes by computed tomography (CT) imaging to be an independent prognostic factor [12]. Due to its improved accuracy, magnetic resonance imaging (MRI) has now virtually replaced CT scan as means of determining the stage of tumors, including NPC, before they are treated [13]. MRI is superior to CT scan for diagnosing the gross extent of tumor infiltration and retropharyngeal lymph node metastasis.

\section{Biomed Central}


In this study, we retrospectively reviewed MRI images in a separate cohort of NPC patients to further validate of the previous finding regarding the use of bidimensional measurement as means of prognosis in NPC. If confirmed to be an independent prognostic factor, then prognostic ability of the current TNM staging approach can be improved.

\section{Methods}

\section{Patient selection}

The method of bidimensional measurement of primary tumor and retropharyngeal nodes (BDMprn) in NPC was derived from a cohort of newly diagnosed NPC patients with definite treatment [12]. All patients had histological confirmed NPC and received CT scan of the nasopharyngeal area, chest X-ray, ultrasound or CT scan of the abdomen and whole body bone scan. All cases were restaged based on criteria outlined in the 6th edition of the AJCC staging system [14] Patients received a complete course of radiotherapy (70 Gy - 75 Gy). Patients who received concurrent chemoradiotherapy (CCRT) received three cycles of cisplatin during the same period that were undergoing radiotherapy. Subsequent adjuvant chemotherapy consisting of cisplatin and 5-FU was arranged as guidelines [2]. Using computed tomography-derived measurement, bidimensional measurement of primary tumor and retropharyngeal nodes (BDMprn) in NPC had good correlation with gross tumor volume (Spearman' correlation coefficient $=0.845, \mathrm{P}<0.001)$. The intrarater reliability for BDM was good. In multivariate analysis, BDMprn was an independent prognostic factor for any relapse [Hazard ratio $(\mathrm{HR})=1.066 ; \mathrm{P}=$ 0.029], and overall survival [HR $=1.097 ; \mathrm{P}=0.007$ ]. NPC patients with large BDMprn conferred a poor survival and more recurrences[12].

Validation of the bidimensional measurement of primary tumor and retropharyngeal nodes was performed using a cohort which included NPC patients treated at Kaohsiung Veterans General Hospital from 2002 to 2009. The means of treating NPC patients in these two hospitals is similar. All patients received a complete course of radiotherapy (70 Gy - 75 Gy). Concurrent chemotherapy was arranged for NPC patients with advanced T (T2-4) classification or positive neck metastasis. Patients with T2b-T4 or N2-3 disease underwent subsequent adjuvant chemotherapy.

Before treatment, all NPC patients received physical examinations, fiberoptic examinations, chest X-rays, ultrasound or CT scan of the abdomen, whole body bone scan and MRI of nasopharyngeal area. Similarly, all cases were restaged according to the AJCC stage classification system, which was modified in 2002.

\section{MRI technique and measurements}

Gross tumor volume of primary tumor and retropharyngeal nodes (GTVprn) of NPC measurement was performed with summation of area technique as described previously [12]. The lateral retropharyngeal nodes were considered malignant if its shortest axial dimension was $5 \mathrm{~mm}$ or greater, and any visible node in the median retropharyngeal group was considered metastatic [15-17]. Bidimensional measurement of primary tumor and retropharyngeal nodes (BDMprn) was performed as described previously [12]. Briefly, BDMprn was obtained by multiplying the maximum diameter of the nasopharyngeal tumor and retropharyngeal nodes by the greatest measurement perpendicular to it (Figure 1). Bidimensional measurement of primary tumor (BDMp) was calculated by multiplying the maximum diameter of the nasopharyngeal tumor by the greatest measurement perpendicular to it [18]. It was sometimes difficult to evaluate the anatomic extent of primary tumor and retropharyngeal node. In such cases, when the outline of tumor was unclear, a radiologist specializing in head and neck cancer helped demarcate the margin. When there was skull base involvement or parapharyngeal space invasion, we could measure the gross tumor and retropharyngeal nodes using the same methods in Figure 1.

The calculation of the three measurements was as the followings:

GTVprn $=\Sigma$ Outlined area of primary tumor and retropharyngeal nodes $\times$ (slice thickness + split interval).

BDMprn $=\Sigma$ Maximum diameter $\times$ greatest perpendicular of primary tumor and retropharyngeal nodes.

$\mathrm{BDMp}=$ Maximum diameter $\times$ greatest perpendicular of primary tumor.

\section{Clinical endpoints}

Clinical endpoints were 3 -year overall survival, any recurrence and distant metastasis. Six weeks after completing the course of treatment, patients received endoscopy and biopsy of the nasopharynx if necessary. Two months after the course of treatment, each patient received a MRI examination. Chest X-rays, abdominal sonography, and whole body bone scan were performed regularly.

\section{Statistics}

Intrarater reliability was measured using the intraclass correlation coefficient. Overall survival, distant metastasis-free survival and recurrence-free survival were calculated according to the methods of Kaplan and Meier. Differences between multiple survival curves were compared using the log-rank test. The prognostic influence of BDM was assessed using Cox proportional hazards 

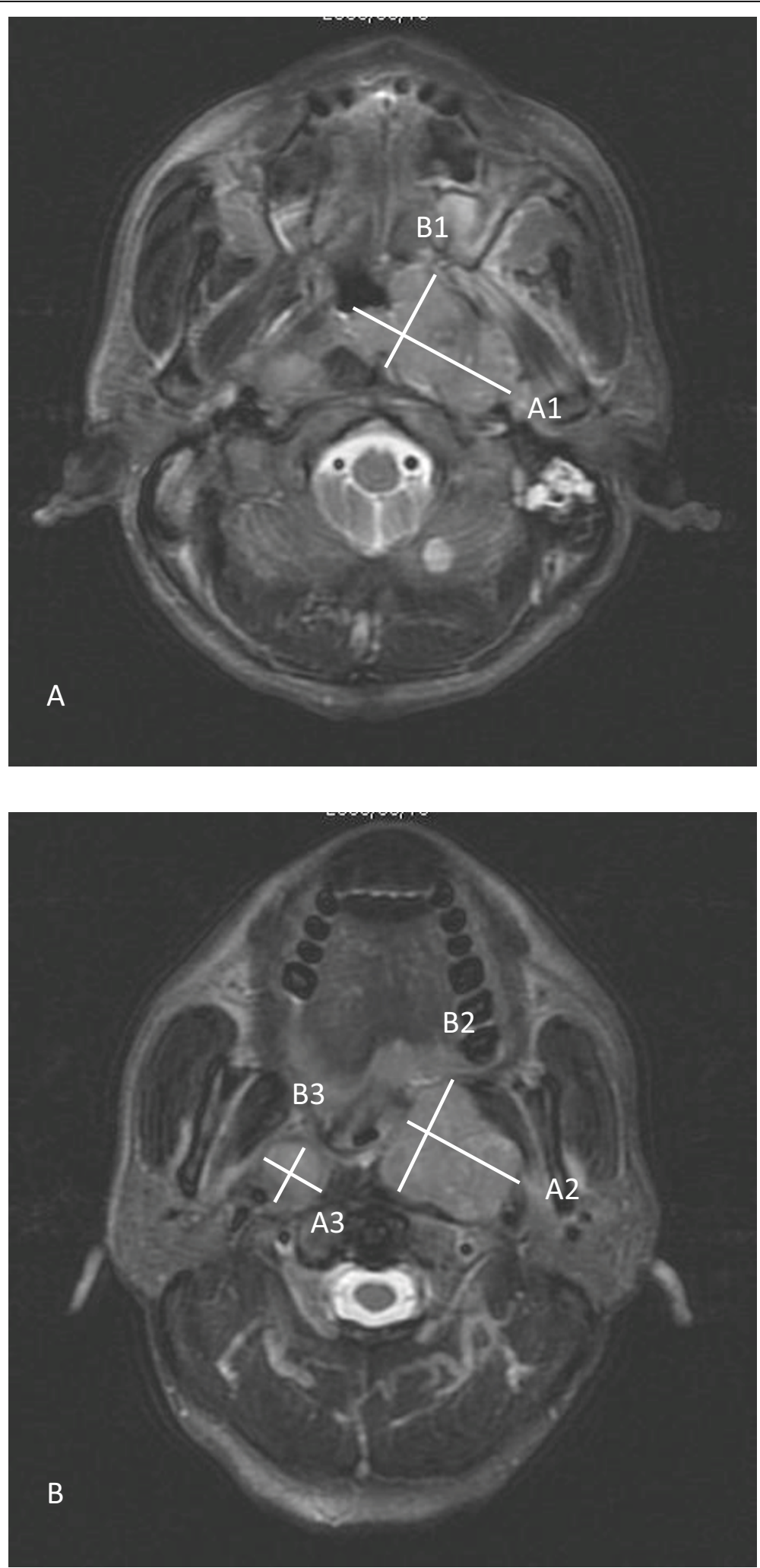

Figure 1 T2-weighted postcontrast MR image in the axial plane. The bidimensional measurement of primary tumor and retropharyngeal nodes (BDMprn) was obtained by summation of multiplying the maximum diameter by the greatest measurement perpendicular to it in nasopharyngeal tumor (A) and retropharyngeal nodes (B). BDMprn $\left(\mathrm{cm}^{2}\right)=A 1 \times B 1+A 2 \times B 2+A 3 \times B 3$. 
multivariate model after adjusting for age, gender, and chemotherapy status. BDM cut-off values were obtained by receiver operating characteristic (ROC) curve analysis. All statistical operations were performed using the Statistical Package for Social Sciences, version 15.0 (SPSS, Chicago, IL).

\section{Results}

\section{Patient and disease characteristics}

The intrarater reliability correlation coefficients for GTVprn, BDMprn, and BDMp were 0.956(0.935-0.97), 0.964 (0.912-0.986), and 0.966 (0.916-0.987). Table 1 shows the characteristics of patients in validation cohort. The mean age was $51 \pm 13$ years. Of the 103 NPC patients, 77 (75\%) patients were men. 88 patients $(85 \%)$ had advanced stage (stage III-IV). These NPC patients were followed up a median of 43 months (range 9-80 months). Thirty-four (33\%) in the validation cohort had recurrences, including 15 (15\%) with locoregional recurrence and $12(12 \%)$ with distant metastasis. Eighteen patients $(18 \%)$ expired. The 3-year overall survival rate

Table 1 Patient Characteristics

\begin{tabular}{lc}
\hline Variables & $\begin{array}{c}\text { Validation cohort }(\boldsymbol{n}=\mathbf{1 0 3}) \\
\text { No. of patients (\%) }\end{array}$ \\
\hline Age (years) & $51 \pm 13$ \\
Mean \pm SD & \\
Gender & $77(75)$ \\
Male & $26(25)$ \\
Female & \\
Stage & $3(3)$ \\
I & $12(12)$ \\
II & $56(54)$ \\
III & $32(31)$ \\
IV & \\
T classification & $25(24)$ \\
T1 & $20(19)$ \\
T2 & $34(33)$ \\
T3 & $24(23)$ \\
T4 & \\
N classification & \\
N0 & $6(6)$ \\
N1 & $11(11)$ \\
N2 & $72(70)$ \\
N3 & $14(14)$ \\
Histology grade & \\
Non-keratinizing carcinoma & $13(13)$ \\
Undifferentiated carcinoma & $90(90)$ \\
Treatment modality & \\
RT/CCRT & $76(74)$ \\
CCRT+CT & $27(26)$ \\
\hline SD, standard devatin;
\end{tabular}

$\mathrm{SD}$, standard deviation; RT, radiotherapy; CCRT, concurrent chemoradiotherapy; CCRT+CT, concurrent chemoradiotherapy with adjuvant chemotherapy was $87 \%$, locoregional control survival rate $88 \%$, distant metastasis-free survival rate $89 \%$, and recurrence-free survival rate $79 \%$.

\section{Univariate and multivariate analysis}

Based on our univariate analysis, bidimensional measurement of primary tumor and retropharyngeal nodes was found to be a significant prognostic factor (Table 2). Adjusting for age, gender, and chemotherapy status, our multivariate analysis found bidimensional measurement of primary tumor and retropharyngeal nodes to significantly predict overall survival $(\mathrm{HR}=1.012$; $95 \%$ CI: 1.014-1.12; $\mathrm{P}=0.012$ ) and metastasis-free survival $(\mathrm{HR}=1.046 ; 95 \% \mathrm{CI}: 1.002-1.121 ; \mathrm{P}=0.042)$. The bidimensional measurement of primary tumor was not a significant predictor for outcomes in multivariate analysis. Both univariate and multivariate analysis found gross tumor volume of primary tumor and retropharyngeal nodes to be a significant prognostic factor.

\section{Bidimensional measurement and risk groups}

We wanted to further validate the prognostic ability of bidimensional measurement of primary tumor and retropharyngeal nodes using MRI findings. After analyzing trade-off, we chose $15 \mathrm{~cm}^{2}$ as the cut-off point in the validation cohort (additional file 1). Using this cut-off point, we further divided validation cohort into a smaller BDMprn group (67\%) and a larger BDMprn group (33\%). The smaller BDMprn group had greater 3-year overall survival, distant metastasis-free survival, and recurrence-free survival rates than the large BDM group. $(92.3 \%$ vs. $73.7 \%, \mathrm{P}=0.009 ; 94 \%$ vs. $75 \%, \mathrm{P}=$ $0.034 ; 64.1 \%$ vs. $59.7 \%, \mathrm{P}=0.082$ ) (Figure. $2 \mathrm{~A}$ and $2 \mathrm{~B}$ ), and they were at lower risk.

\section{Discussion}

In a previous study, CT-derived bidimensional measurement of primary tumor and retropharyngeal nodes could be used to predict prognosis of NPC [12]. Using MRI to validate the ability of bidimensional measurement of primary tumor and retropharyngeal nodes to predict NPC outcomes in a validation cohort, we found BDMprn remained an independent prognostic factor for overall survival as well as metastasis-free survival. Adopting a BDMprn of $15 \mathrm{~cm}^{2}$ as cut-off point in validation cohort, NPC patients whose BDMprn was less than $15 \mathrm{~cm}^{2}$ had a better 3-year overall survival rate and distant metastasis-free survival rate than those with BDMprn above this cut off point. Based on these two studies, we have found that BDMprn can be used to stratify patients into two different prognostic groups with significantly different overall survival and metastatic rates. 
Table 2 Univariate and multivariate analysis results $(n=103)$

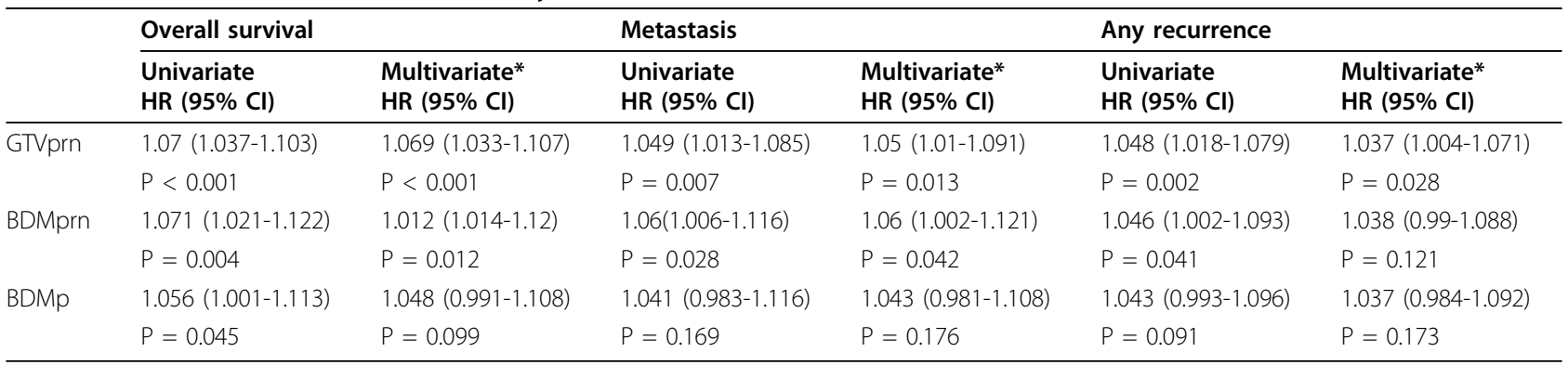

GTVprn, gross tumor volume of primary tumor and retropharyngeal nodes; BDMprn, bidimensional measurement of primary tumor and retropharyngeal nodes; $\mathrm{BDMp}$, bidimensional measurement of primary tumor; $\mathrm{HR}$, hazard ratio; $95 \% \mathrm{Cl}, 95 \%$ confidence interval.

*Multivariate analysis: adjusted for age, gender, and chemotherapy status.

Although the current TNM staging system for NPC is widely used, it has been reported to have several deficiencies. Mao et al. [3] and Cheng et al. [4] have not found any significant differences in local-relapse free survival among the T1, T2, and T3 NPC subgroups. Recently, gross tumor volume has been reported to be a risk factor for local recurrence of NPC $[5,6,19]$. However, measurement of gross tumor volume is time-consuming, and the technology, expertise, and manpower are often not available in routine clinical practice.

In a study of bidimensional and unidimensional MRIderived measurement to reflect NPC tumor anatomic extent at diagnosis or the change in size after treatment, King et al. [18] found that BDM of primary tumor was a quicker and more widely applicable method than tumor volume measurement and that it could be used to assess tumor response. However, measurement of retropharyngeal nodes were not included in that series. Tang et al. [20] showed that retropharyngeal lymph node metastasis affects the distant metastasis-free survival rates of NPC, and Wang et al. [21] found a good correlation between retropharyngeal lymph node metastasis and parapharyngeal space involvement as well as metastasis to Level II, III, IV and/or V nodes. Based on these findings, our previous study modified the approach used by King et al. to include both primary tumor and retropharyngeal lymph nodes measurements in our definition of BDM. Previous study found a very close relationship between CT-derived BDMprn, gross tumor volume of primary tumor and retropharyngeal nodes, and overall survival [12]. In the present study, also incorporating retropharyngeal lymph node measurements, we found MRI-derived BDMprn could also predict overall survival as well as metastasis free survival in NPC patients.

In our study, we found that we could use BDMprn to categorize patients into low- and high-risk groups. This distinction would facilitate treatment decisions, as it would spare low-risk NPC patients from receiving aggressive treatment. Although NPC is markedly radiosensitive, there is a high failure rate in treatment due to its metastatic behavior. Improvement in the outcome for NPC relies on the delivery of higher radiation doses [22]. While radiotherapy is the only standard treatment for early-stage NPC (stage I), the combination of cisplatin-based chemotherapy and radiotherapy is used to treat patients with advanced NPC (stage II-IVB) [2]. The latter group not only receives higher doses of radiotherapy, they also receive chemotherapy, both associated with significant comorbidity, including myocardial infarction, severe nutrient deficiency, nephrotoxicity, transverse myelitis, leukopenia, and central nervous system disease $[2,23,24]$. Recent study revealed that NPC patients with GTVprn $\geqq 13 \mathrm{ml}$ conferred a poor prognosis and may benefit from $\geqq 4$ cycles of chemotherapy [25]. This series implied that high-risk NPC patients, such as large GTVprn, could benefit from more intensive chemotherapy and radiotherapy. The treatment goals for NPC is to adjust chemoradiotherapy dosages to achieve adequate anticancer effects without overly increasing the development of such complications. It would be important and valuable if high-risk NPC patients could be identified in order to adjust the radiation dose and tailor chemotherapy protocol. In this way, high-risk patients (larger BDMprn) may benefit from more extensive treatment approaches, such as more intensive chemotherapy or higher dose of radiation, whereas low-risk patients (smaller BDMprn) may do well with standard therapy and can be spared the severe toxic side effects of more radical therapy.

\section{Conclusion}

We have validated bidimensional measurement of primary tumor and retropharyngeal nodes in a different cohort of NPC with pretreatment staging by MRI. BDMprn, derived by MRI, is closely related to survival rates and metastatic rates of NPC patients. BDMprn can stratify patients into two different prognostic groups with significantly different overall survival. Nasopharyngeal carcinoma patients with large bidimensional 


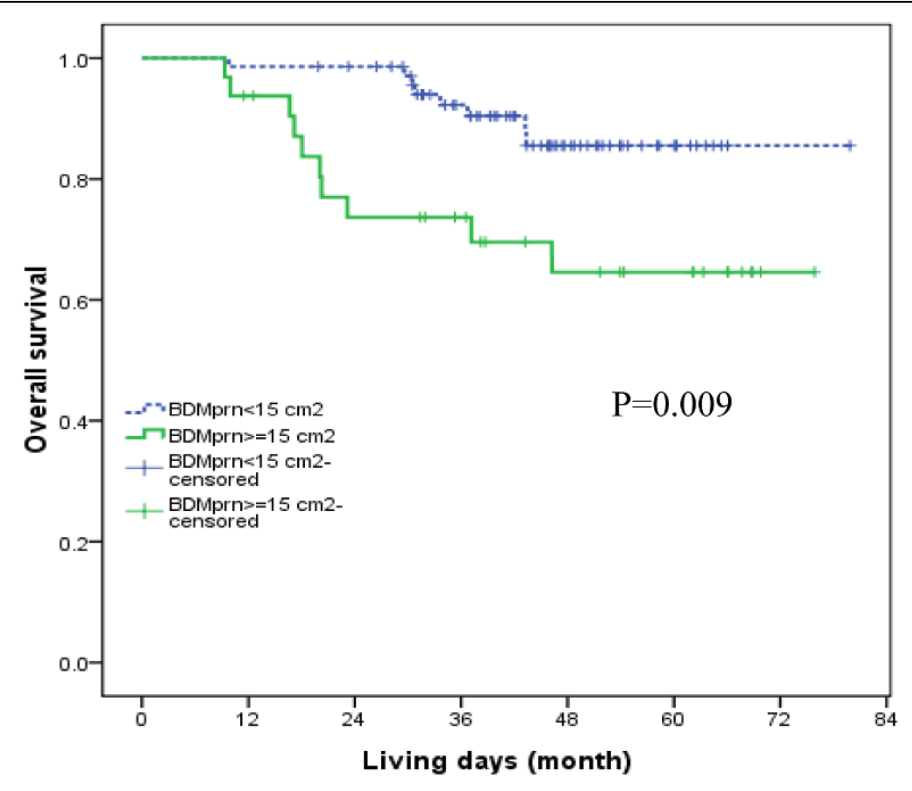

A

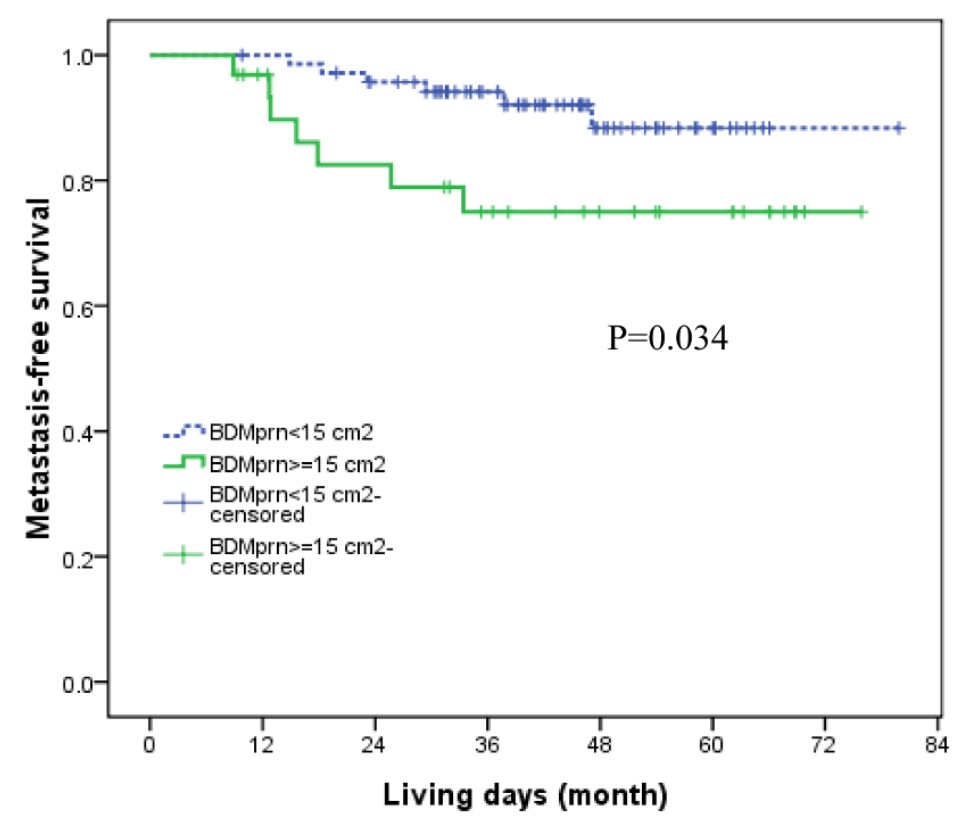

$\mathrm{B}$

Figure 2 Survival curves. (A)Probability of overall survival rates by small versus large BDMprn. (B) Probability of distant metastasis-free survival rates by BDMprn. 
measurement have poor survival rates and high metastasis potential. BDMprn might be used in the future for the design of clinical trials, the prediction of survival, and treatment decisions.

\section{Additional material}

Additional file 1: Table S1. Validity of BDMprn using death, distant metastasis or any recurrence as the standard.

\section{Author details}

'Department of Otolaryngology, Kaohsiung Veterans General Hospital, Kaohsiung, Taiwan. ${ }^{2}$ Department of Otolaryngology, Buddhist Tzu Chi Dalin General Hospital, Chiayi County 622, Taiwan. ${ }^{3}$ School of Medicine, Tzu Chi University, Hualian, Taiwan.

\section{Authors' contributions}

TSC and CCL designed the study, collected the data, interpreted the results of the study, and oversaw the project completion. STC, YYH, KPC and CCC participated in preparing of data acquisition. TSC and CCL performed the statistical analysis and drafted the manuscript. All authors contributed to the scientific setup of the study and revised the manuscript critically, and they have approved the final version of the manuscript.

\section{Competing interests}

The authors declare that they have no competing interests.

Received: 11 June 2010 Accepted: 16 August 2010

Published: 16 August 2010

\section{References}

1. Department of Heath, The Executive Yuan: Cancer registry annual report, Republic of China, 2001. 2004

2. Al-Sarraf M, LeBlanc M, Giri PG, Fu KK, Cooper J, Vuong T, Forastiere AA, Adams G, Sakr WA, Schuller DE, Ensley JF: Chemoradiotherapy versus radiotherapy in patients with advanced nasopharyngeal cancer: phase III randomized Intergroup study 0099. J Clin Oncol 1998, 16:1310-1317.

3. Mao YP, Xie FY, Liu LZ, Sun Y, Li L, Tang LL, Liao XB, Xu HY, Chen L, Lai SZ, et al: Re-evaluation of 6th edition of AJCC staging system for nasopharyngeal carcinoma and proposed improvement based on magnetic resonance imaging. Int J Radiat Oncol Biol Phys 2009, 73:1326-1334.

4. Cheng SH, Tsai SY, Horng CF, Yen KL, Jian JJ, Chan KY, Lin CY, Terng SD, Tsou MH, Chu NM, et al: A prognostic scoring system for locoregional control in nasopharyngeal carcinoma following conformal radiotherapy. Int J Radiat Oncol Biol Phys 2006, 66:992-1003.

5. Sze WM, Lee AW, Yau TK, Yeung RM, Lau KY, Leung SK, Hung AW, Lee MC, Chappell R, Chan K: Primary tumor volume of nasopharyngeal carcinoma: prognostic significance for local control. Int J Radiat Oncol Biol Phys 2004, 59:21-27.

6. Chu ST, Wu PH, Chou P, Lee CC: Primary tumor volume of nasopharyngeal carcinoma: prognostic significance for recurrence and survival rate. Eur Arch Otorhinolaryngol 2008, 265(Suppl 1):S115-120.

7. Chen MK, Chen TH, Liu JP, Chang CC, Chie WC: Better prediction of prognosis for patients with nasopharyngeal carcinoma using primary tumor volume. Cancer 2004, 100:2160-2166.

8. Chua DT, Sham JS, Kwong DL, Tai KS, Wu PM, Lo M, Yung A, Choy D, Leong $L$ : Volumetric analysis of tumor extent in nasopharyngeal carcinoma and correlation with treatment outcome. Int I Radiat Oncol Biol Phys 1997, 39:711-719.

9. World Health Organization: WHO handbook for reporting results of cancer treatment Geneva Albany, N.Y: World Health Organization; sold by WHO Publications Centre USA 1979.

10. Miller $A B$, Hoogstraten $B$, Staquet $M$, Winkler A: Reporting results of cancer treatment. Cancer 1981, 47:207-214.
11. Therasse P, Arbuck SG, Eisenhauer EA, Wanders J, Kaplan RS, Rubinstein L, Verweij J, Van Glabbeke M, van Oosterom AT, Christian MC, Gwyther SG: New guidelines to evaluate the response to treatment in solid tumors. European Organization for Research and Treatment of Cancer, National Cancer Institute of the United States, National Cancer Institute of Canada. J Natl Cancer Inst 2000, 92:205-216.

12. Lee CC, Ho HC, Su YC, Lee MS, Hsiao SH, Hwang JH, Hung SK, Chou P: Bidimensional measurement of nasopharyngeal carcinoma: a simple method to predict outcomes. Clin Otolaryngol 2009, 34:26-33.

13. Chung NN, Ting LL, Hsu WC, Lui LT, Wang PM: Impact of magnetic resonance imaging versus $\mathrm{CT}$ on nasopharyngeal carcinoma: primary tumor target delineation for radiotherapy. Head Neck 2004, 26:241-246.

14. AJCC cancer staging handbook New York: Springer 2002.

15. Ichimura K: Can Rouviere's lymph nodes in non-malignant subjects be identified with MRI? Auris Nasus Larynx 1993, 20:117-123.

16. Watarai J, Seino Y, Kobayashi M, Shindo M, Kato T: CT of retropharyngeal lymph node metastasis from maxillary carcinoma. Acta Radiol 1993, 34:492-495.

17. King AD, Ahuja AT, Leung SF, Lam WW, Teo P, Chan YL, Metreweli C: Neck node metastases from nasopharyngeal carcinoma: MR imaging of patterns of disease. Head Neck 2000, 22:275-281.

18. King AD, Zee B, Yuen EH, Leung SF, Yeung DK, Ma BB, Wong JK, Kam MK, Ahuja AT, Chan AT: Nasopharyngeal cancers: which method should be used to measure these irregularly shaped tumors on cross-sectional imaging? Int J Radiat Oncol Biol Phys 2007, 69:148-154.

19. Shen C, Lu JJ, Gu Y, Zhu G, Hu C, He S: Prognostic impact of primary tumor volume in patients with nasopharyngeal carcinoma treated by definitive radiation therapy. Laryngoscope 2008, 118:1206-1210.

20. Tang L, Li L, Mao Y, Liu L, Liang S, Chen Y, Sun Y, Liao X, Tian L, Lin A, et al: Retropharyngeal lymph node metastasis in nasopharyngeal carcinoma detected by magnetic resonance imaging: prognostic value and staging categories. Cancer 2008, 113:347-354.

21. Wang XS, Hu CS, Ying HM, Zhou ZR, Ding JH, Feng Y: Patterns of retropharyngeal node metastasis in nasopharyngeal carcinoma. Int J Radiat Oncol Biol Phys 2009, 73:194-201.

22. Perez CA, Devineni VR, Marcial-Vega V, Marks JE, Simpson JR, Kucik N: Carcinoma of the nasopharynx: factors affecting prognosis. Int J Radiat Oncol Biol Phys 1992, 23:271-280.

23. Erkal HS, Serin M, Cakmak A: Nasopharyngeal carcinomas: analysis of patient, tumor and treatment characteristics determining outcome. Radiother Oncol 2001, 61:247-256.

24. Teo PM, Leung SF, Chan AT, Leung TW, Choi PH, Kwan WH, Lee WY, Chau RM, Yu PK, Johnson PJ: Final report of a randomized trial on altered-fractionated radiotherapy in nasopharyngeal carcinoma prematurely terminated by significant increase in neurologic complications. Int J Radiat Oncol Biol Phys 2000, 48:1311-1322.

25. Lee CC, Huang TT, Lee MS, Hsiao SH, Lin HY, Su YC, Hsu FC, Hung SK: Clinical application of tumor volume in advanced nasopharyngeal carcinoma to predict outcome. Radiat Oncol 2010, 5:20.

doi:10.1186/1748-717X-5-72

Cite this article as: Chang et al:: Validation of bidimensional measurement in nasopharyngeal carcinoma. Radiation Oncology 2010 $5: 72$.

\section{Submit your next manuscript to BioMed Central and take full advantage of:}

- Convenient online submission

- Thorough peer review

- No space constraints or color figure charges

- Immediate publication on acceptance

- Inclusion in PubMed, CAS, Scopus and Google Scholar

- Research which is freely available for redistribution 